\title{
The Hadronic Final State in Diffraction at HERA
}

\author{
Riko Wichmann*† (on behalf of the $\mathrm{H} 1$ and ZEUS Collaborations) \\ DESY, Notkestr. 85, 22607 Hamburg, Germany \\ E-mail: 'wichmann@mail.desy.dei
}

ABSTRACT: An extensive study of the hadronic final state in diffractive deep inelastic scattering (DIS) at HERA is presented. The hadronic final state was investigated by the $\mathrm{H} 1$ and ZEUS collaborations in terms of inclusive particle distributions, dijet and three-jet production, and compared to various Monte Carlo models. Most aspects of the final state topology are described by models assuming a resolved Pomeron dominated by gluons.

\section{Introduction}

Recent measurements from the H1 and ZEUS collaborations have shown that, in QCDinspired models of diffraction, the Pomeron $(\mathbb{P})$ can be described as an object with its

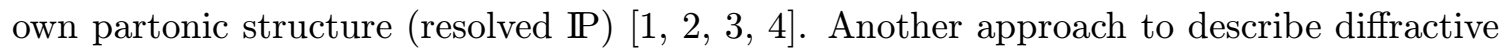
scattering, which is implemented in models, is based on the dissociation of the virtual photon. In these models, the photon dissociates into $q \bar{q}$ or $q \bar{q} g$ which couple to the proton via the exchange of a gluon ladder [i-j. The simplest implementation of a net color singlet

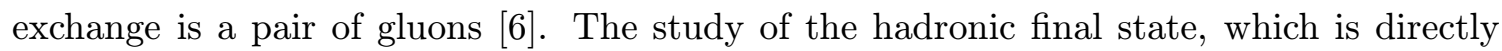
related to the parton configuration, is therefore a natural place to learn more about the underlying dynamics of diffractive DIS.

The diffractive DIS process $e p \rightarrow e^{\prime} X Y$, where $X$ represents the observed multihadron final state of a photon-dissociated system with an invariant mass $M_{X}$, is characterized by the presence of a fast forward-scattered baryonic state $Y$. This baryonic state $Y$ can be identified as a proton when it is detected by specially designed detectors such as the ZEUS

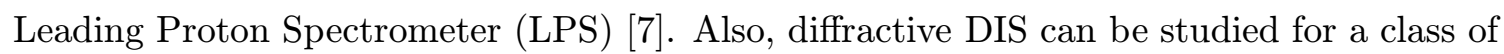
events in which a large rapidity gap (LRG) between the observed hadronic final state $X$ and the proton beam direction is identified. Under these conditions, the baryonic state $Y$ is constrained to have low mass.

${ }^{*}$ Speaker.

${ }^{\dagger}$ also supported by: Pennsylvania State University, University Park, PA 16803, USA 


\section{Inclusive Particle Distributions}

The ZEUS collaboration has presented results based on $13.8 \mathrm{pb}^{-1}$ of data collected in 1997 on the global properties of the hadronic final state $X$ in the center-of-mass (CMS) frame of the $\gamma^{*} \mathbb{P}$ system [i⿱乛龰] $]$. The results are given in the kinematic range $4<M_{X}<35 \mathrm{GeV}$, $4<Q^{2}<150 \mathrm{GeV}^{2}, 0.0003<x_{\mathbb{P}}<0.03$, and $70<W<250 \mathrm{GeV}$, where $Q^{2}$ is the negative squared four-momentum transfer to the virtual photon, $x_{\mathbb{P}}$ the fraction of the proton's longitudinal momentum transferred to the $\mathbb{P}$, and $W$ the center-of-mass energy of the $\gamma^{*} p$ system. Diffractive events were selected by measuring the scattered proton with the LPS and requiring that the proton carries at least $97 \%$ of the initial beam energy.

The average $p_{T}^{2}$ of particles as a function of $x_{F}$, the fractional longitudinal momentum of the particle to the CMS energy, referred to as seagull plots, are shown in Fig. 高 in three bins of $M_{X}$ and compared with predictions from three Monte Carlo (MC) models: RAPGAP [īin, SAT-

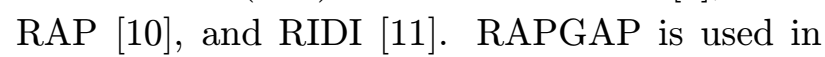
its resolved $\mathbb{P}$ mode based on the $\mathrm{H} 1$ fit 2 to the diffractive structure function $F_{2}^{D(3)}$ [通. SATRAP [1] $\left.{ }_{1}^{0}\right]$ is an implementation of the saturation model (by Golec-Biernat and Wüsthoff) [1] $[1 \overline{2}]$ into the RAPGAP framework and produces $q \bar{q}$ and $q \bar{q} g$ parton final states in the approach of photon dissociation. The RIDI generator, which is based

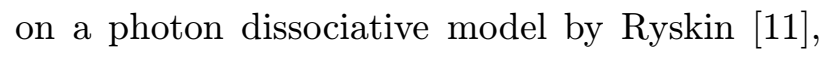
also includes $q \bar{q}$ and $q \bar{q} g$ final states. The data exhibit an asymmetry with larger average $p_{T}^{2}$ in the photon direction which becomes more apparent as $M_{X}$ increases. RAPGAP and RIDI produce roughly the correct asymmetric $p_{T}$ while the

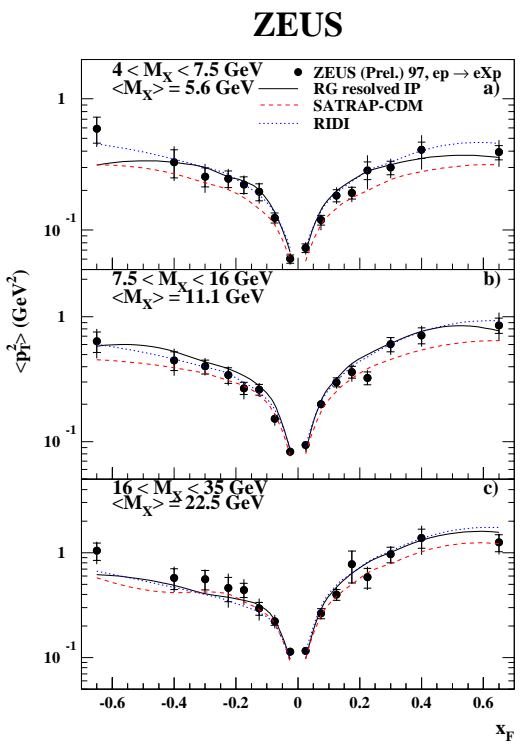

Figure 1: Average squared transverse momentum as a function of $x_{F}$ (seagull plot) in three bins of $M_{X}$ in the $\gamma^{*} \mathbb{P}$ center-ofmass system. Positive $x_{F}$ is in the direction of the virtual photon. transverse momentum generated by SATRAP is smaller than in the data. In all models, the asymmetry is produced at the patron level by the presence of a gluon in the $\mathbb{P}$ direction and a $q \bar{q}$ in the $\gamma^{*}$ hemisphere.

\section{Jet Production}

MC Models, which correctly describe the measurement discussed in the previous section, produce predominately a three parton final state. It is therefore a natural step to extend the analysis of global event properties to the study of jet production.

\subsection{Dijet Production}

Properties of diffractive dijets were studied by the $\mathrm{H} 1$ collaboration using $18.0 \mathrm{pb}^{-1}$ of data collected from 1996 to 1997 in the kinematic range $4<Q^{2}<80 \mathrm{GeV}^{2}, x_{\mathbb{P}}<0.05$, 
$p_{T, \text { jet }}>4 \mathrm{GeV}$ and $0.1<y<0.7$ using LRG events [1] ${ }_{1}$ in, where $y$ is the the DIS inelasticity variable. The analysis was performed in the CMS frame of the $\gamma^{*} p$ system using the CDF cone jet algorithm [i] $\left.{ }_{1}^{1} \bar{i}\right]$ with a cone radius of $R=1$. Events with at least two jets were selected for the dijet sample.

In Fig. '2i, the differential dijet cross section $d \sigma / d z_{\mathbb{P}}^{(j e t s)}$ as a function of $z_{\mathbb{P}}^{(j e t s)}=\left(Q^{2}+M_{12}^{2}\right) /\left(Q^{2}+M_{X}^{2}\right)$ is shown in four intervals of the scale $\mu^{2}=Q^{2}+p_{T}^{2}$. In resolved $\mathbb{P}$ models, the variable $z_{\mathbb{P}}$ can be interpreted as the fraction of the pomeron momentum carried by the parton (usually gluon) entering the hard scattering that produces the two jets with invariant mass $M_{12}$. Fig. $\underset{2}{2}$ shows that only a small fraction of the $\mathbb{P}$ momentum enters into the hard scattering. Also shown in Fig. ${ }_{1}^{2} \overline{2}$ are predictions from the RAPGAP MC generator using the $\mathrm{H} 1$ fits 2 and 3 . The MC predictions contain contributions from both direct and resolved virtual photons, where the internal partonic structure in the resolved photon case is taken from the SAS-2D [1] $\left.{ }_{1}^{1} \overline{1}\right]$ parameterization. The dijet data are especially sensitive in the region of large $z_{\mathbb{P}}$ to the shape of the gluon distribution and favor the flatter distribution (H1 fit 2). The $\mathbb{P}$ parton distribution functions are evolved according to the DGLAP equations, which describe well the scale dependence of the diffractive data.

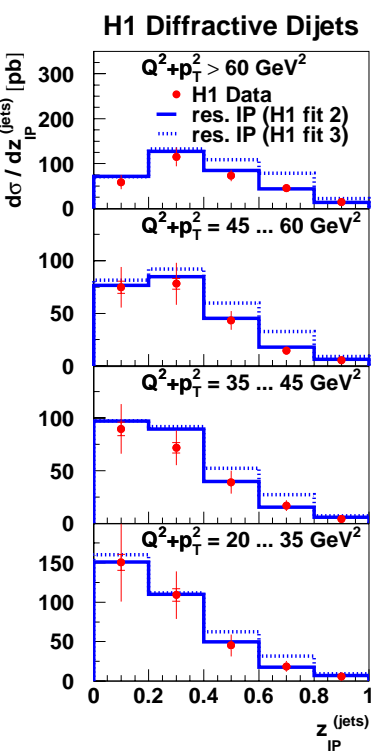

Figure 2: Diffractive dijet cross sections as a function of $z_{\mathbb{P}}$, shown in four intervals of the scale $\mu^{2}=Q^{2}+p_{T}^{2}$.

The picture of a resolved virtual photon can also be interpreted as an approximation to next-to-leading-order (NLO) QCD diagrams or contributions without strong $k_{T}$-ordering. Possible non- $k_{T}$-ordered contributions are investigated using the diffractive differential dijet cross section as a function of $z_{\mathbb{P}}^{(j e t s)}$ in the restricted kinematic range of $x_{\mathbb{P}}<0.01$ (Fig. $\underline{3}_{1}^{\overline{1}} \mathrm{c}$ ). The cross section is compared with predictions from SATRAP, which assumes $k_{T}$-ordering, and from the BJLW model for $q \bar{q}$ production $\left[\overline{1} \overline{1} \overline{\sigma_{1}}\right]$ and for $q \bar{q} g$ production [i] $\overline{1} \overline{1}]$ for two values of the $p_{T}$ cut-off for the gluon. The
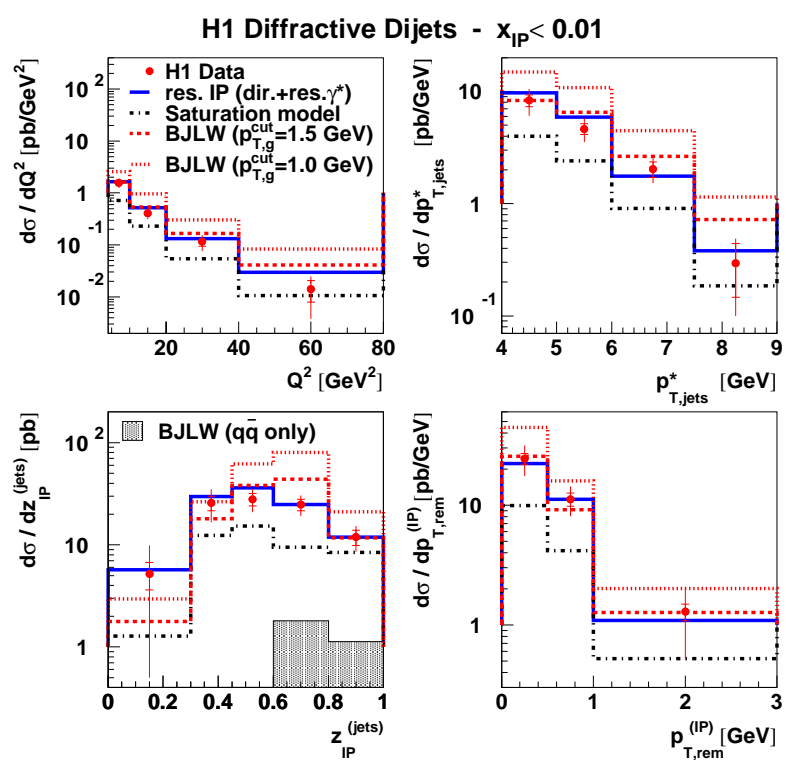

BJLW calculations take non- $k_{T}$-ordered Figure 3: Diffractive dijet cross section in the restricted contributions into account. The conkinematic range $x_{\mathbb{P}}<0.01$. tribution from $q \bar{q}$ in the BJLW model is negligible except at large values of $z_{\mathbb{P}}^{(j e t s)}$ while the data have a significant fraction of 
events also at low values. SATRAP approximately describes the shape of the distribution, however the normalization is too small by a factor of 2 . On the other hand, the production of $q \bar{q} g$ according to the BJLW model has approximately the correct normalization with an appropriately chosen cut-off value for the transverse momentum of the gluon $\left(p_{T, g}^{2}>1.0 \mathrm{GeV}^{2}\right)$.

\subsection{Three-Jet Events}

The comparison of all results with models presented so far shows that the best description of the data is achieved with models which have a dominant contribution from gluons originating either from a gluon-dominated $\mathbb{P}$ or from the dissociation of the virtual photon into $q \bar{q} g$. In all models, the gluon usually travels in the direction of the $\mathbb{P}$. The properties of such a gluon jet can be studied by investigating the production of three jets in diffractive DIS.

The ZEUS collaboration studied three-jet production using $42.74 \mathrm{pb}^{-1}$ of data collected during 1998-2000 in the kinematic region $23<M_{X}<$ $40 \mathrm{GeV}, 200<W<250 \mathrm{GeV}, x_{\mathbb{P}}<0.25$, and $5<Q^{2}<100 \mathrm{GeV}^{2}$ [i] $1 \overline{8} \bar{i}$. Diffractive events were selected by requiring a LRG in the outgoing pro-

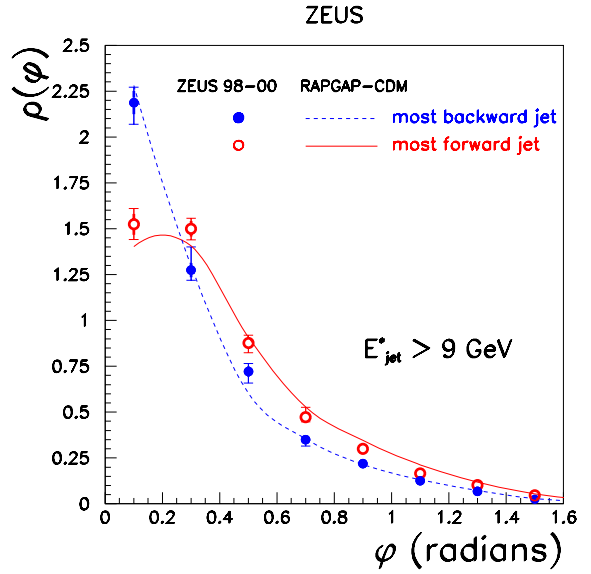

Figure 4: Differential jet shapes, $\rho(\phi)$, for the most-forward and most-backward jets with $E_{\text {jet }}>9 \mathrm{GeV}$ in three-jet events, where the $\mathbb{P}$ defines the forward direction. ton direction. Jets were searched for in the CMS frame of the hadronic system $X$ using the exclusive $k_{T}$-algorithm (Durham algorithm) [i] $\left.1 \overline{1} \overline{9}\right]$. The clustering procedure was repeated until the threshold value $y_{c u t}=0.05$ was exceeded. For the $M_{X}$ region considered, the energy of the resulting jets is $E_{\text {jet }}>4 \mathrm{GeV}$.

Fig. $\overline{4}$ i shows the differential jet shapes, $\rho(\phi)=\sum \Delta E_{\text {jet }}(\phi \pm \delta \phi / 2) /\left(\delta \phi N_{\text {jets }} E_{\text {jet }}\right)$, for the most-forward ( $\mathbb{P}$ direction) and most-backward jet in three-jet events with $E_{\text {jet }}>9 \mathrm{GeV}$. Here, $\Delta E_{\text {jet }}(\phi \pm \delta \phi / 2)$ denotes the sum of all hadronic activity belonging to a given jet whose angular distance to the jet axis is within the range $(\phi-\delta \phi, \phi+\delta \phi)$ for $\delta \phi=0.2$, and $N_{\text {jets }}$ is the number of jets. The jet in the $\mathbb{P}$ direction is broader than the jet in the photon direction. This is well reproduced by models in which the gluon is produced in the direction of the $\mathbb{P}$ and a quark in the photon direction. The observed difference in jet shapes can therefore be attributed to the different nature of the outgoing partons travelling in the $\mathbb{P}$ and $\gamma^{*}$ directions.

\section{Conclusions}

An extensive study of the final state in diffractive DIS in terms of inclusive particle production and jet production at HERA was presented.

Various MC models were used to predict the topology of the hadronic final state in diffractive DIS. The diffractive exchange is modeled either by assuming a resolved $\mathbb{P}$ with 
partonic structure or a photon dissociative approach. The resolved $\mathbb{P}$ model gives the overall best description. The photon dissociative models usually reproduce the shape of the distributions correctly but with a normalization too low by roughly a factor of 2 . Both approaches, however, suggest that at the parton level, the final state in diffractive DIS is dominated by gluons either originating from a gluon-dominated $\mathbb{P}$ (preferring a "flat" gluon distribution which evolves according to the DGLAP equations) or from $q \bar{q} g$ production in photon dissociation. The gluon is produced in the direction of the $\mathbb{P}$.

Comparison of the data with models which do or do not assume strong- $k_{T}$-ordering on the parton level suggests that some extra source of $k_{T}$ (for example, non- $k_{T}$-ordering or including NLO QCD terms) might be necessary to produce a better description of the data.

\section{References}

[1] ZEUS Collaboration, J. Breitweg et al., Eur. Phys. J. ZEUS Collaboration, J. Breitweg et al., Eur. Phys. J. C 6 (1999) 43.

[2] H1 Collaboration, C. Adloff et al., Eur. Phys. J.

[3] H1 Collaboration, C. Adloff et al., 'Phys. Lett. B $\mathbf{4 2 8} 81998) 206$.

[4] H1 Collaboration, C. Adloff et al., 'Z. Physik C $\mathbf{7} 6$ (1997) 613."

[5] J. Bartels, et al., 'Ëur. Phys. J. C 7 (1999) 4431.

[6] F. Low, 'P S. Nussinov, Phys. Rev. Lett. 34 (1975) 1286!

[7] ZEUS Collaboration, M. Derrick et al., Z. Physik C.

[8] ZEUS Collaboration, S. Chekanov et al., accepted by Phys. Rev. D, DESY-01-097.

[9] RAPGAP: H. Jung, Comput. Phys. Commun 86 (1995) 147.

[10] H. Kowalski, Proceedings of the Ringberg Workshop "New Trends in HERA Physics", Ringberg, June 1999, DESY 99-141.

[11] RIDI 2.0: M.G. Ryskin and A Solano, Proceedings of the Workshop on Monte Carlo Generators for HERA Physics, DESY 1998/99, DESY-PROC-1999-02 (1999) 386 and references therein.

[12] K. Golec-Biernat and M. Wüsthoff, Phys. Rev. D 60 0 1999$) 114023$.

[13] H1 Collaboration, C. Adloff et al., Eur. Phys. J. C 20 (2001) 29.

[14] CDF Collaboration, F. Abe et al., 'Physs. Rev. D $45-(1992) 1448$

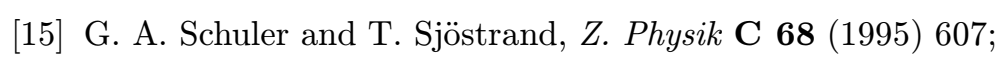

G. A. Schuler and T. Sjöstrand,

[16] J. Bartels, H. Lotter and M. Wüsthoff, Phys. Lett. B 379 1996$) 2399_{i}^{\prime}$

J. Bartels, et al., 'P $\underline{P}$ hs. Lett. B $\mathbf{3} 86 \mathbf{6}(1996) 389$.

[17] J. Bartels, H. Jung and M. Wüsthoff, 'Eur. Phys. J. C 11 (1999) 111'.

[18] ZEUS Collaboration, S. Chekanov et al., 'P Phys. Lett. B $\mathbf{5 1 6} 6(2001) 273$

[19] S. Catani et al., 'Phys. Lett. B $26 \mathbf{9} 9(1991) 432$. 